\title{
latrogenic factors contributed to the high rate of preterm birth in a community hospital
}

\author{
Lu Huang ${ }^{1 \#}$, Zhong Chen ${ }^{1 \#}$, Jiawen $\mathrm{Li}^{1}$, Yuanyuan Chen ${ }^{1}, \mathrm{Ke} \mathrm{Yin}^{1}$, Yu Chen ${ }^{1}$, Lingqing Hu${ }^{1}$, \\ Xiaomin Zheng ${ }^{1}$, Tao Zhou ${ }^{1}$, Yunlong Zhu ${ }^{1}$, Daozhen Chen ${ }^{1}$, Nanbert Zhong ${ }^{2}$ \\ ${ }^{1}$ Department of Obstetrics, The Affiliated Wuxi Maternity and Child Health Care Hospital of Nanjing Medical University, Wuxi, China; ${ }^{2}$ New York \\ State Developmental Disorders Basic Research Institute, Staten Island, NY, USA \\ Contributions: (I) Conception and design: L Huang, D Chen, N Zhong; (II) Administrative support: D Chen, L Hu; (III) Provision of study materials \\ or patients: Y Chen, K Yin, Y Chen; (IV) Collection and assembly of data: Z Tao, J Li, Y Zhu; (V) Data analysis and interpretation: X Zheng, J Li; (VI) \\ Manuscript writing: All authors; (VII) Final approval of manuscript: All authors. \\ \#These authors contributed equally to this work. \\ Correspondence to: Prof. Daozhen Chen. Department of Obstetrics, The Affiliated Wuxi Maternity and Child Health Care Hospital of Nanjing Medical \\ University, No. 48 Huaishu Lane, Liangxi District, Wuxi 214002, China. Email: chendaozhen@163.com; Prof. Nanbert Zhong. Director, New York State \\ Institute for Basic Research in Developmental Disabilities, 1050 Forest Hill Road, Staten Island, NY, 10314, USA. Email: nanbert.zhong@opwdd.ny.gov.
}

\begin{abstract}
Background: Preterm birth (PTB) rates have been increased significantly in recent years, mostly due to obstetric intervention. This study presents the incidence of PTB in community hospitals by assessing the association between pregnancy complications and iatrogenic PTB.

Methods: A total of 6,693 pregnancies were enrolled in the Wuxi Maternity and Child Health Care Hospital of Nanjing Medical University. They were divided into two groups ( $<35$ and $\geq 35$ years of age) to examine the effect of maternal age on PTB. Binary logistic and multiple linear regression analyses were used to assess the relationship between PTB and pregnancy complications.

Results: This study provided the incidence of PTB and found that PP, PROM, and ICP increased the risk of PTB, indicating that pregnancy complications have led to the iatrogenic PTB and contributed to the high rate of PTB, especially in the group of advanced-age pregnant women. The prevalence of PTB was $9.53 \%$. Placenta previa (PP), premature rupture of membranes (PROM), and intrahepatic cholestasis of pregnancy (ICP) were significantly associated with PTB. Among all the risk factors, hypertension, disease in pregnancy, premature PROM, and PP were observed as independent key factors for iatrogenic PTBs. In the advancedage group, PP and pPROM increased the risk of PTB.

Conclusions: It is often necessary to terminate a pregnancy in community hospitals to balance the safety of the fetus and the maternal comorbid symptoms, which has led to nosocomial premature delivery. Therefore, high-risk pregnancies should be carefully evaluated and comprehensively treated with caution to balance the preterm rate and the safety of the pregnant woman and fetus, and the pros and cons of the outcomes, which has brought a challenge to an obstetrician to reduce the proportion of iatrogenic PTB.
\end{abstract}

Keywords: Preterm birth (PTB); pregnancy complications; premature rupture of membranes (PROMs)

Submitted Sep 05, 2021. Accepted for publication Oct 20, 2021.

doi: $10.21037 / \mathrm{tp}-21-458$

View this article at: https://dx.doi.org/10.21037/tp-21-458

\section{Introduction}

Preterm birth (PTB), resulting from multiple etiological factors, is the leading cause of neonatal mortality worldwide and the second common cause of mortality for under-five children (1). The World Health Organization (WHO) defined PTB as the live birth delivered before 37 weeks of gestation or born too soon at less than 259 days from the first date of a woman's last menstrual period (LMP). 
Annually, there are 15 million PTB worldwide, and China has been listed as the second highest number of PTB in the world (2), with an incidence of about $7.2 \%$ $(3,4)$ with a $14 \%$ increase annually $(5)$. Recently, the rate of PTB in China has increased slightly to $9.63 \%$ in 2016 (6). There has been an enormous negative impact on family and society between PTB and its associated sequelae (7-9). Based on the "Born Too Soon: the global action report on preterm birth" in 2010, with the global average PTB rate of $11.1 \%, 15$ million babies were born, and China had more than 1.1 million PTB with a rate of $7.1 \%(2,10)$. However, the reported PTB rate could be a variation in different studies here in China. According to 107,905 live births in 39 hospitals from 14 provinces, the rate of PTB was $7.1 \%$ in 2011 (11).

Due to the Chinese report in 2015, the reason for the under-5 years old child mortality and the second cause of child death after congenital abnormalities are the PTBrelated complications. They create more than one million deaths per year $(12,13)$. PTB may lead to short- or longterm morbidities, affecting children's health development, leading to blindness, deafness, neurodevelopmental delay, psychiatric disturbances, diabetes, or chronic noncommunicable disease in adulthood $(14,15)$.

Due to the changes in social awareness, vocational priority, maternal education $(16,17)$, and society stress nowadays (18-20), advanced maternal age is reportedly associated with the upraised risk for PTB (21); there is no clear relationship between pregnancy complications and PTB (22). Elderly maternal has been defined as pregnant women over the age of 35 and having their first baby, a phenomenon now common and almost universal in The Organization for Economic Co-operation and Development (OECD) countries. From 1990 to 2004, the prevalence of advanced maternal age increased by $9 \%(23-26)$ in the United Kingdom. Advanced maternal age is associated with decreased fertility and fecundity in pregnant women, resulting in adverse perinatal birth outcomes. Stillbirth, miscarriage, fetal growth restriction, PTB, and pregnancy complications of preeclampsia (PE) and gestational diabetes mellitus (GDM) were increases by the advanced maternal age (25). As important covariates in the study of adverse birth outcomes, the effect of maternal age on the association between PTB and pregnancy complications remains unknown. Risk factors associated with PTB have been divided into three clusters: maternal, fetal, and placental conditions, and are characterized as the major contributors to iatrogenic PTBs in China (26).
This study aimed to evaluate the association between iatrogenic PTB and pregnancy complications from a population distribution perspective, and further investigate if advanced maternal age potentially affected this association. We present the following article in accordance with the STROBE reporting checklist (available at https:// dx.doi.org/10.21037/tp-21-458).

\section{Methods}

\section{Population studied}

We collected birth data from 2014 to 2016 at the Wuxi Maternity and Child Health Care Hospital of Nanjing Medical University for this retrospective cohort study. All procedures performed in this study involving human participants were in accordance with the Declaration of Helsinki (as revised in 2013). The study was approved by the Research Ethical Committee of Nanjing Medical University (No. 2019-02-0102-01) and informed consent was taken from all the patients.

A total of 6,693 pregnancies were enrolled. We retrieved birth registries to create data about mothers and neonates [e.g., maternal age, family address, gestational age, delivery date, delivery mode (vaginal birth and cesarean selection), birth weight, and neonatal gender]. The inclusion criteria included women who were residents of Wuxi, those with singleton live births, and those with maternal age with delivery years. Women with infants with birth weight $<500$ $\mathrm{g}$ were excluded from the study because these infants could not survive (27). Multiple exclusion criteria may apply to a single birth. Medical records and information were reviewed by trained research nurses using the WHO Global Survey on Maternal and Perinatal Health and the WHO MultiCountry Survey on Maternal and Neonatal Health $(28,29)$.

\section{Study design}

PTB was divided into spontaneous PTB [following preterm premature rupture of membranes (PROMs), preterm labor, or cervical insufficiency] or iatrogenic (medically indicated) PTB (cesarean section or induction of labor due to maternal/fetal indications) based on the etiology (30,31). Iatrogenic PTB may result from pathological pregnancy, including maternal and fetal indications. Maternal indications include hypertensive disorders in pregnancy (HDP, including pregnancy-induced hypertension, PE/ eclampsia, chronic hypertension superimposed by $\mathrm{PE}$ ), 
placenta previa (PP), diabetes mellitus or GDM, renal and cardiac disease, immune system disease, thyroid disease and asthma, history of miscarriage or stillbirth, history of PTB, and conception by assisted reproductive technology (ART). Fetal conditions include fetal distress, multiple pregnancies, fetal anomalies, sex indetermination, and antepartum stillbirth. Delivery age, parity (nulliparous or multiparous), delivery mode (virginal birth or cesarean birth), mode of fertilization (natural conception or assisted reproductive technique), PROMs, infant gender (male or female), and year of birth (from 2014 to 2016) data was collected. This study collected the clinical information of maternal and child conditions from the hospital record registry. Two gestation-related complications were included in the analyses as binary variables. PTB and advanced age were also considered important confounders. Identifying risk factors for PTB may help guide clinicians and obstetric management for pregnant women, to help community hospitals comprehensively consider the safety of the fetus and mother, balance the safety of the fetus and whether the mother can continue the pregnancy, thus reduce the rate of iatrogenic PTB in community hospitals.

\section{Statistical analysis}

Data were weighted to consider the study design and other aspects of the sampling. We used the Bootstrap method to calculate the $95 \%$ confidence intervals (CIs) of PTB incidence. Maternal age was grouped into child-bearing age ( $<35$ years of age) and advanced maternal age ( $\geq 35$ years of age) groups. Based on the WHO BMI classification, maternal pregnancy body mass index (BMI) was classified as underweight $\left(<18.5 \mathrm{~kg} / \mathrm{m}^{2}\right)$, normal $\left(18.5-24.9 \mathrm{~kg} / \mathrm{m}^{2}\right)$, and overweight or obese $\left(\geq 25 \mathrm{~kg} / \mathrm{m}^{2}\right)$ (32). Binary logistic regression and multiple linear regression analyses were used in this study. We examine the relationships between PTB and pregnancy complications (maternal, fetal conditions). The various risk factors were analyzed in the multiple linear regression model, with gestational age as the continuous dependent variable. The binary logistic regression model assessed the association between PTB and pregnancy complications. Year of birth, gestational age, and the number of weeks are included in the model to estimate the relationship with the model and to adjust the model. The participants were also grouped according to their maternal age to assess the association between pregnancy complications and PTB. Between the groups, a student t-test was used to find the homogeneity of variance. One-way
ANOVA and chi-square test were used for comparison in three or more groups.

The statistically significant for $\mathrm{P}$ value $<0.05$ and highly significant for $\mathrm{P}$ values $<0.01$ were considered differences. We evaluated the underlying causes of PTB in SPSS version 22.0 (SPSS Inc., Chicago, IL, USA).

\section{Results}

The patient clinical characteristics are shown in Table 1. As shown in Table 1, the prevalence of PTB was $9.53 \%$, while pregnant women with GDM, gestational hypertension, ART, and advanced age accounted for $1.25 \%, 0.19 \%$, $0.31 \%$, and $1.49 \%$ respectively. The number of women with PTB who were primiparous was more than twice that of women with PTB with a fertility history. The distribution of delivery mode (vaginal birth and cesarean selection) was significantly different $(\mathrm{P}<0.01)$. Meanwhile, the gender of the delivered infants was roughly equal. There was only one case of pregnancy with maternal underweight BMI. 36.36\% of cases were normal weight, and $63.47 \%$ were overweight or obese.

Pregnancy complications are shown in Table 2, including maternal diseases of GDM, gestational hypertension (HYP), intrahepatic cholestasis of pregnancy (ICP), pregnancy with thyroid dysfunction (THY), pregnancy with hepatitis B virus (HEP), PP, and premature rupture of fetal membrane (PROM). We found a perplexing problem: in pregnancy complication diseases, cross-correlation was not high, especially among many common clinical diseases (PP and GDM). Except for PROM and pregnancy complications, there was no significant clinical crosscorrelation. Meanwhile, it was interesting to find that PTB and HYP, PTB and ICP, PTB and PP had some slight clinical relevance. It was maybe that severe hypertension and ICP, PP with vaginal bleeding often choose iatrogenic termination of pregnancy before 37 weeks.

Pregnancy complications include GDM, HYP, ICP, THY, HEP, PP, and preterm premature rupture of fetal membrane $<37$ weeks (pPROM). The correlations between the pregnancy complications in PTBs are shown in Table 3.

We found that if a premature rupture of the membrane occurs, the possibility of PTB is higher when there are two types of pregnancy complications, including one pregnant woman with both complications of HYP and PP. Simultaneously, it was interesting to find that PPROM and multiple pregnancy complications have a higher correlation, while a single pregnancy complication has no significant 
Table 1 Distribution of the study population in Wuxi, China [2014-2016]

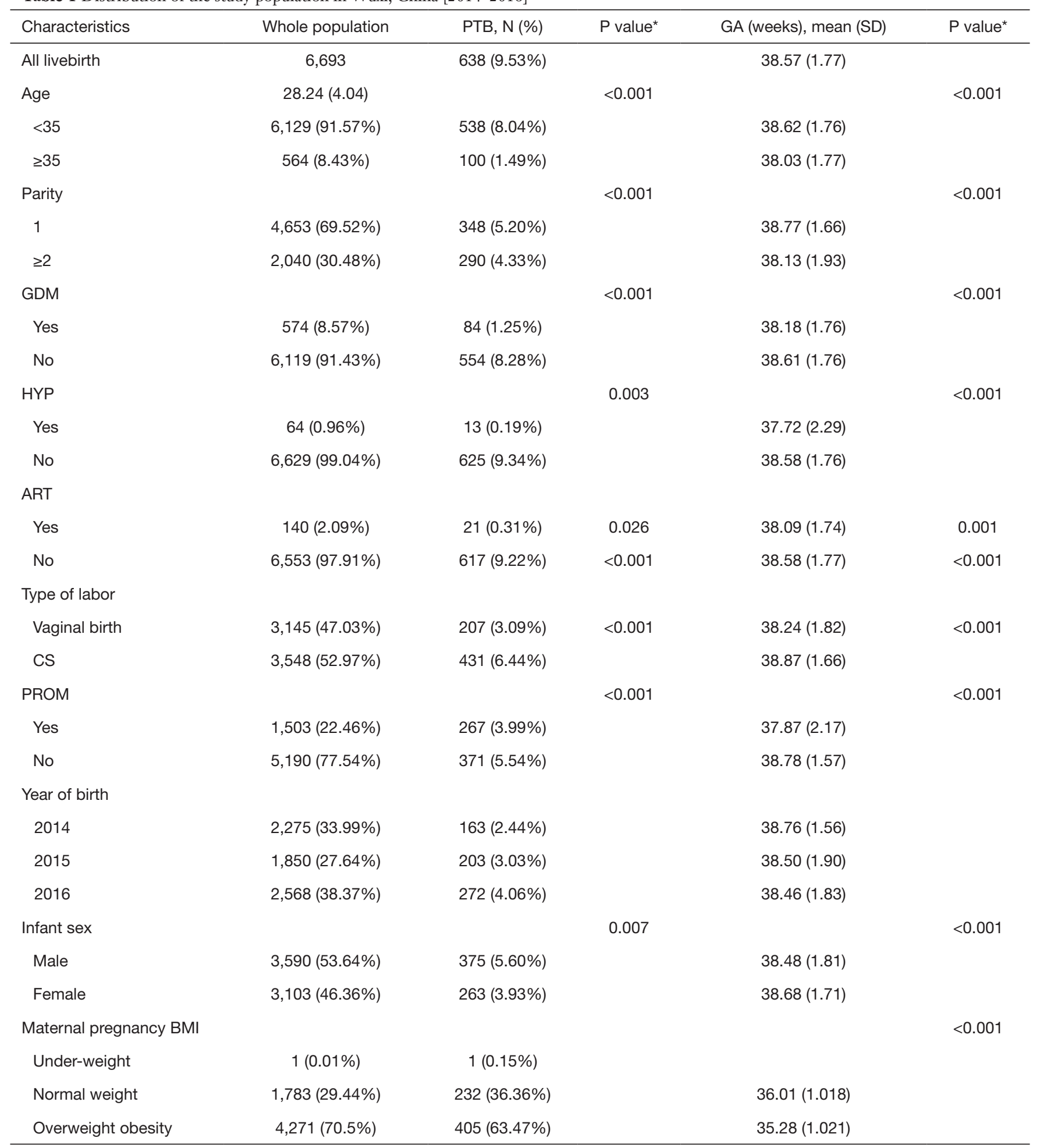

*, P value <0.05 by two-side deemed statistically significant. SD, standard deviations; PTB, preterm birth; GA, gestational age; GDM, gestational diabetes mellitus; HYP, gestational hypertension; ART, assisted reproductive technology; CS, cesarean section; PROM, premature rupture of (fetal) membrane; BMI, body mass index. 
Table 2 Correlation of pregnancy complications

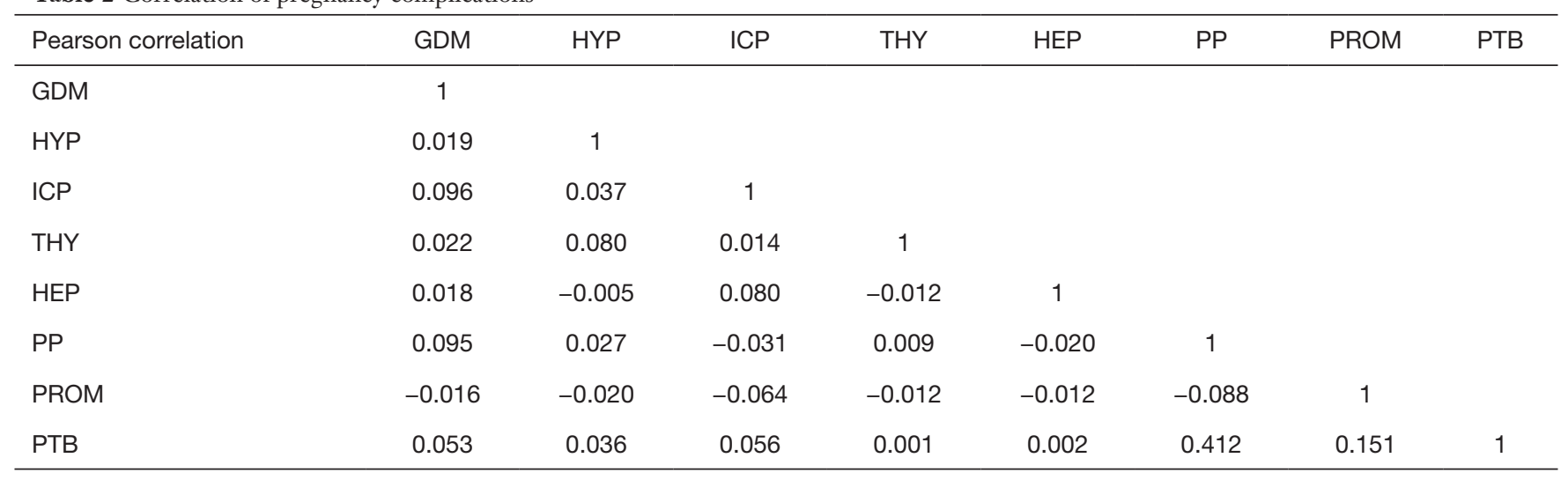

GDM, gestational diabetes mellitus; HYP, gestational hypertension; ICP, intrahepatic cholestasis of pregnancy; THY, pregnancy with thyroid dysfunction; HEP, pregnancy with HBV carrying; PP, placenta Previa; PROM, premature rupture of fetal membrane.

Table 3 Correlation between pPROM and pregnancy complication in PTB

\begin{tabular}{|c|c|c|c|c|c|c|c|}
\hline Pearson correlation & GDM & HYP & $\mathrm{ICP}$ & THY & HEP & PP & pPROM \\
\hline HYP & 0.042 & 1 & & & & & \\
\hline ICP & 0.176 & 0.057 & 1 & & & & \\
\hline THY & 0.063 & 0.028 & 0.031 & 1 & & & \\
\hline PP & 0.096 & 0.043 & -0.161 & 0.058 & -0.062 & 1 & \\
\hline pPROM & -0.077 & -0.100 & -0.146 & -0.080 & 0.020 & -0.479 & 1 \\
\hline
\end{tabular}

GDM, gestational diabetes mellitus; HYP, gestational hypertension; ICP, intrahepatic cholestasis of pregnancy; THY, pregnancy with thyroid dysfunction; HEP, pregnancy with HBV carrying; PP, placenta Previa; pPROM, preterm premature rupture of fetal membrane $<37$ weeks.

clinical correlation.

The odds ratios (ORs) with 95\% CIs for correlation of PTB with age-adjusted and clinical characteristics were shown in Table 4.

The stratification analysis revealed a significant association between PTB and BMI (OR $=15.615$; $95 \%$ CI: 12.275-19.864). In child-bearing women, parity and PTB were significantly associated (OR $=2.061$; 95\% CI: 0.999 4.251), and pPROMs and PTB were significantly associated $(\mathrm{OR}=0.227$; 95\% CI: 1.615-3.071). Moreover, labor and PTB were significantly associated $(\mathrm{OR}=2.870 ; 95 \%$ CI: 1.942-4.243). Meanwhile, PTB was not significantly associated with GA, gender, or IBW (individual birth weight). After adjusting for age, BMI was significantly associated with PTB in both the advanced-age and childbearing age groups. In the advanced-age group, parity and labor increased the risk of PTB by 3.2\% (95\% CI: 1.496$2.011 \%$ ) and $4.3 \%$ (95\% CI: $2.403-2.899 \%)$, respectively. Moreover, pPROMs were significantly associated with PTB in the advanced-age and child-bearing age groups. However, we found that PPROM increased the risk for PTB by $3.9 \%$ (95\% CI: $1.641-3.130 \%$ ) in advanced-age women. In the event of PPROM in advanced-age women, the risk of PTB increases, which may be related to psychological factors and cervical function.

In the sensitivity analysis, PTB and pregnancy complications were associated with age (Table 5). The association between PTB and PP, PROMs, and ICP was assessed in child-bearing and advanced-maternal age groups. The stratification analysis revealed a significant association between PTB and PP (OR $=17.502$; 95\% CI: 14.197-21.576). GDM was significantly associated with 
Table 4 Correlation of preterm birth with age adjustment and clinical characteristics

\begin{tabular}{|c|c|c|c|c|c|c|}
\hline Clinical characteristic & \multicolumn{3}{|c|}{ Preterm birth without age adjustment } & \multicolumn{3}{|c|}{ Preterm birth with age adjustment } \\
\hline BMI & 15.615 & $12.275-19.864$ & $<0.001$ & 16.717 & $13.533-20.650$ & $<0.001$ \\
\hline GA & 1.722 & $0.795-1.423$ & 0.678 & 1.077 & $0.803-1.445$ & 0.621 \\
\hline Parity & 2.061 & $0.999-4.251$ & 0.049 & 1.735 & $1.496-2.011$ & $<0.001$ \\
\hline Sex & 0.978 & $0.581-1.646$ & 0.934 & 0.997 & $0.623-1.596$ & 0.991 \\
\hline IBW & 1.061 & $0.685-1.644$ & 0.790 & 1.001 & $0.670-1.495$ & 0.996 \\
\hline pPROM & 2.227 & $1.615-3.071$ & $<0.001^{*}$ & 2.266 & $1.641-3.130$ & $<0.001$ \\
\hline
\end{tabular}

*, $\mathrm{P}$ value $<0.05$. BMI, body mass index; GA, gestational age; IBW, birth weight; parity, nulliparous or multiparous; labor, vaginal birth or cesarean birth; pPROM, preterm premature rupture of (fetal) membrane <37 weeks; OR, odds ratio; Cl, confidence interval.

Table 5 Correlation of preterm birth with age adjustment and pregnancy complications

\begin{tabular}{|c|c|c|c|c|c|c|}
\hline Pregnancy complications & \multicolumn{3}{|c|}{ Preterm birth without age adjustment } & \multicolumn{3}{|c|}{ Preterm birth with an age adjustment } \\
\hline PP & 17.502 & $14.197-21.576$ & $<0.001$ & 16.778 & $13.563-20.755$ & $<0.001$ \\
\hline GDM & 1.722 & $1.345-2.205$ & $<0.001$ & 1.540 & $1.198-1.980$ & $<0.001$ \\
\hline HYP & 2.449 & $1.352-4.527$ & 0.004 & 2.191 & $1.177-4.079$ & 0.013 \\
\hline THY & 1.010 & $0.632,1.613$ & 0.967 & 0.987 & $0.617-1.579$ & 0.958 \\
\hline HEP & 1.040 & $0.698,1.550$ & 0.848 & 1.001 & $0.670-1.495$ & 0.996 \\
\hline PROM & 2.806 & $2.369-3.323$ & $<0.001$ & 2.830 & $2.287-3.354$ & $<0.001$ \\
\hline pPROM & 2.227 & $1.615-3.071$ & $<0.001$ & 2.266 & $1.641-3.130$ & $<0.001$ \\
\hline
\end{tabular}

PP, placenta previa; GDM, gestational diabetes mellitus; HYP, gestational hypertension; ICP, intrahepatic cholestasis of pregnancy; THY, pregnancy with thyroid dysfunction; HEP, pregnancy with HBV carrying; PROM, premature rupture of (fetal) membrane; pPROM, preterm premature rupture of (fetal) membrane $<37$ weeks; OR, odds ratio; $\mathrm{Cl}$, confidence interval.

PTB in both the advanced-age and child-bearing age groups (OR $=1.722 ; 95 \% \mathrm{CI}: 1.345-2.205)$. In child-bearing women, pPROMs and PTB were significantly associated $(\mathrm{OR}=2.227 ; 95 \%$ CI: 1.615-3.071). Moreover, ICP and PTB were significantly associated $(\mathrm{OR}=2.275 ; 95 \%$ CI: 1.583-3.270). Meanwhile, PTB was not significantly associated with gestational hypertension, pregnancy with thyroid dysfunction, or pregnancy with HBV infection.

After stratification analysis, PP was significantly associated with PTB in both the advanced-age and childbearing age groups. In the advanced-age group, PP and pPROMs increased the risk of PTB by $8 \%(95 \%$ CI: $13.563-20.755)$ and $6 \%$ (95\% CI: 1.641-3.130), respectively. Moreover, in the advanced-age group, pPROMs were significantly associated with PTB. ICP was significantly associated with PTB in the advanced-age and child-bearing age groups. However, ICP was found to correlate significantly with premature delivery in advancedage women, while in women of child-bearing age, ICP has a significant correlation with preterm delivery. The rate of PTB decreased by $1.3 \%$ (95\% CI: $1.587-3.293 \%$ ). The occurrence of ICP in the advanced-age group was rare, and it was unclear that the association effect of age between ICP and PTB.

Figure $1 \mathrm{~A}$ demonstrates $\mathrm{PTB}$ subgroups: iatrogenic $\mathrm{PTB}$ and spontaneous PTB. Iatrogenic PTB has the largest 
A

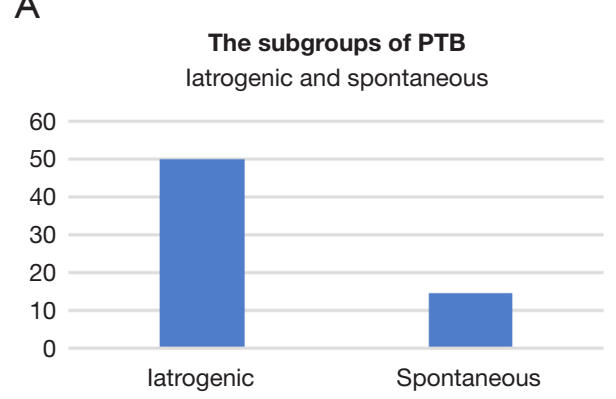

C

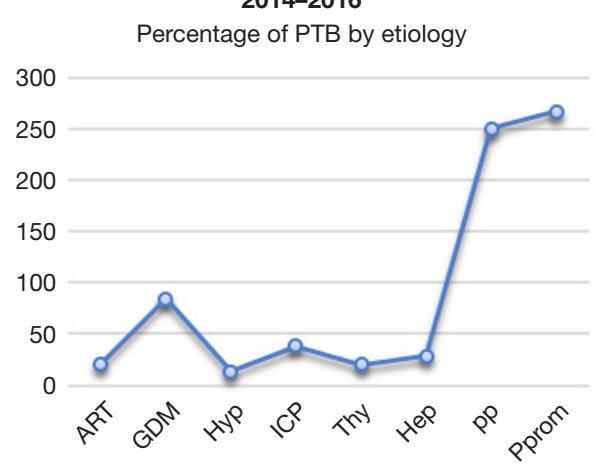

$E$

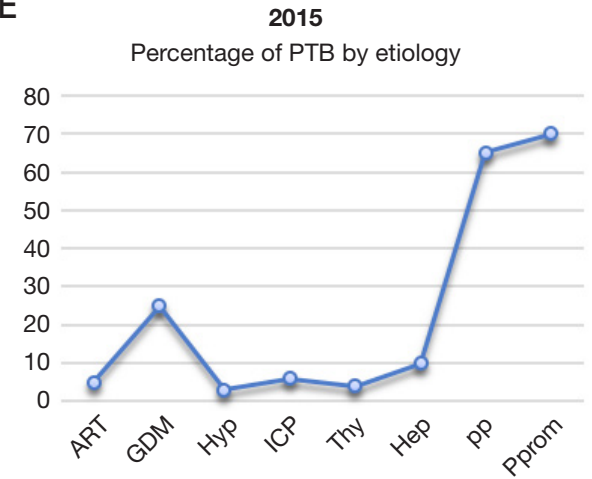

B

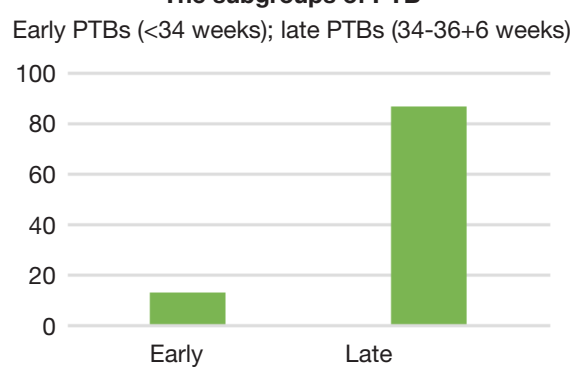

D

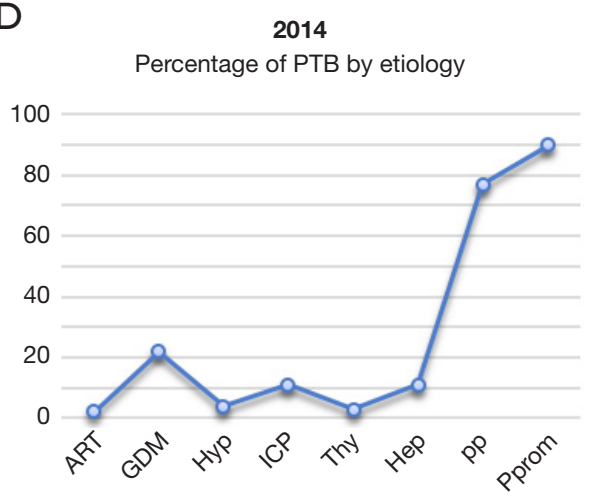

F

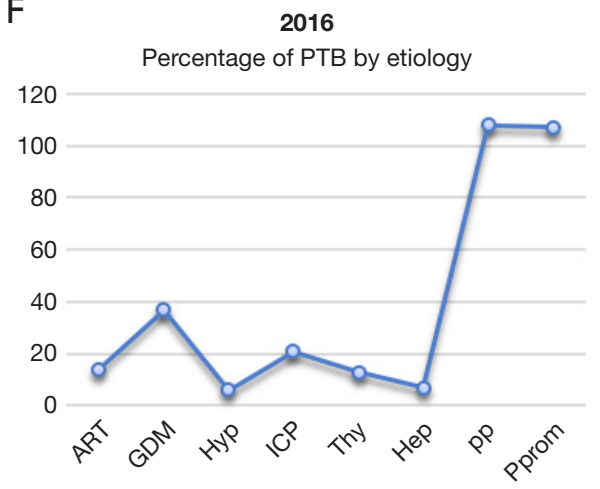

Figure 1 Percentage of preterm births by subgroups and etiology. PTB, preterm birth; ART, assisted reproductive technology; GDM, gestational diabetes mellitus; HYP, gestational hypertension; ICP, intrahepatic cholestasis of pregnancy; THY, pregnancy with thyroid dysfunction; HEP, pregnancy with HBV carrying; PP, placenta Previa; pPROM, preterm premature rupture of (fetal) membrane $<37$ weeks.

proportion of PTB (49.84\%), including spontaneous onset of labor $(14.42 \%)$ and pPROMs (41.8\%). Figure $1 B$ shows that early PTBs ( $<34$ weeks) and late PTBs (34-36+6 weeks) accounted for $13.16 \%$ and $86.83 \%$. To be a higher proportion of early PTB and late PTB in iatrogenic PTB than spontaneous PTB $(57.14 \%$ and $6.23 \%$, respectively). Risk factors associated with iatrogenic PTB, categorized into two major clusters-maternal and fetal conditions, were the major contributors to iatrogenic PTB in China.
Figure $1 C$ presents the percentage of PTBs by etiology from 2014-2016: ART (3.29\%), GDM (13.16\%), HYP (2.03\%), ICP (5.95\%), THY (3.13\%), HEP (4.38\%), PP (39.18\%), and pPROM (41.8\%). Figure $1 D-1 F$ show that among all the risk factors, HYP, pPROM, and PP were the three key factors for iatrogenic PTBs.

The correlation between pregnancy comorbidities and delivery methods in 6693 pregnant women in China is shown in Figure 2. There were 2,959 complications and 


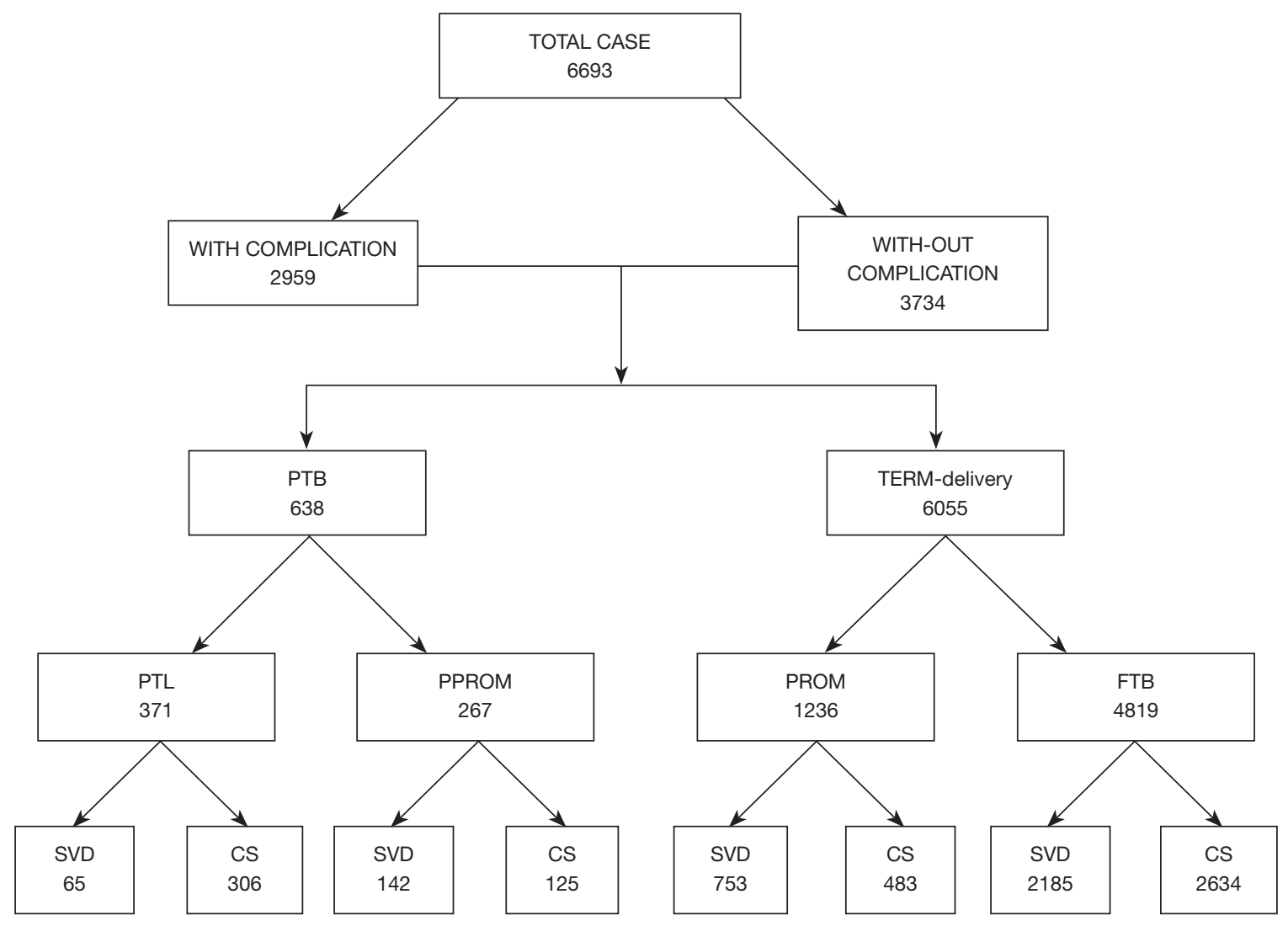

Figure 2 Correlation of pregnancy with or without complication and delivery mode. SVD, standard vaginal delivery; CS, cesarean section; PTB, preterm birth; PTL, preterm birth labor; PPROM, preterm premature rupture of (fetal) membrane <37 weeks; PROM, premature rupture of (fetal) membrane; FTB, full term delivery of membrane.

3,734 without complications. The prevalence of PTB was $638 / 6,693(9.53 \%)$, and the term delivery rate was $6,055 / 6,693$ (90.4\%). In the PTBs, a cesarean section accounted for $67.5 \%$; it accounted for $51.4 \%$ in the term deliveries. From this figure, the rate of iatrogenic PTB had a significant association with the delivery mode, especially a cesarean section. If the rate of cesarean sections can be lowered, the rate of iatrogenic PTB would be decreased, and consequently, the total number of PTBs would be reduced.

\section{Discussion}

The prevalence of PTB was $9.53 \%$ in the cohort of the study, of which $49.84 \%$ were iatrogenic PTB in China. By binary logistic regression and multiple linear regression analyses, we found that early ( $<34$ weeks), and late (34-36+6 weeks), PTBs accounted for $13.16 \%$ and
$86.83 \%$ of PTBs, respectively, and that the proportion of iatrogenic PTB was greater than spontaneous PTB (57.14\% and $6.23 \%$, respectively). We also assessed the effect of pregnancy complications and gestational age, and found that pregnancy complications were associated with PTB and gestational age. Among women with or without advanced maternal age, those with GDM, HYP, and pregnancy with HBV infection had no risk for PTB, and those with PP, PROM, and ICP were significantly associated with PTB.

It is well-known that PTB is a complex syndrome and has many underlying multiple mechanisms. Our results were consistent with previous studies that PTB has many risk factors (33). Since premature birth is an independent factor related to adverse neonatal outcomes, clinicians and health care providers should fully evaluate highrisk pregnancies where PTB may occur and implement appropriate interventions $(34,35)$. Concerning the prevention and management of these maternal and fetal 
conditions, the top priority for future research to reduce the PTB rate was a balance of benefits and risks which favors cesarean delivery over expectant management. Because different methods are used to calculate the PTB rate, the incidence of PTB is very inconsistent. Different countries define premature birth as 20 or 28 weeks to 36 plus 6 weeks. In China, PTB is legally defined as 28 to $36+6$ weeks, so previous studies may underestimate the incidence of PTB (5). In addition, the incidence of PTB may also be affected by the accuracy of gestational age estimates. Ultrasound estimation of gestational age in early pregnancy provides an excellent choice (36). If the estimate of gestational age is corrected by early ultrasound examination, the PTB rate will be accurately estimated.

This study found that PP and pPROM with advanced maternal age had an increased risk for PTB compared with those $<35$ years of age. Given the pronounced pregnancy trend at increased ages, PP, and pPROM, high attention should be concerned to the health of pregnant women and their pregnancy outcomes. PTB is usually analyzed based on gestational age at delivery and clinical manifestations $(36,37)$. Higher average maternal age, more frequent use of assisted reproductive ARTs, increase in non-infertilityrelated multiple gestations, and higher rates of preterm inductions and cesarean deliveries, were considered to contribute to the increasing PTB rate (38). Acknowledge that these risks have led to reducing non-medically indicated late PTBs and changes in the management of some pregnancy complications favoring a later delivery timing $(39,40)$. Among all the risk factors, HYP, pPROM, and PP were observed as the independent key factors for iatrogenic PTBs. In the advanced-age group, PP and pPROM increased the risk of PTB. It will be important to prevent PTB and reduce late PTB in the future by understanding pregnancy complications that have caused this shift in the directionality of trends in the rate of preterm delivery over the past decade.

Women of advanced age may be at risk for PP or PROM. Similarly, it was easy to understand that advanced maternal age may increase the number of pregnancies in their lives. Nowadays, many highly educated mothers have a comfortable social position and postpone the age of delivering a child, especially in developed countries. Women of advanced age are mostly residents of developed cities and have an increased risk for adverse perinatal birth and neonate outcomes, perhaps due to the high incidence of maternal complications (41) (e.g., GDM, PE, and HYP). In the advanced-age group, PP and PROM increased the risk of PTB. Maternal, fetal, and placental conditions account for nearly $66 \%$ of all preterm newborns, while unknown etiology accounts for $33 \%$. In addition, we found that in women of child-bearing age, ICP was significantly associated with PTB. However, after adjusting for age, the risk decreased, possibly because ICP in women with higher age was rare; the gestational age effect of ICP and PTB remained unclear.

From the findings of this research, we conclude that the rate of iatrogenic PTB is significantly associated with the delivery method, especially for a cesarean section. It has been demonstrated PTB associates with previous cesarean sections, with the higher the number of previous cesarean sections, the higher the risk of PTB (42). Pregnant women with iatrogenic premature birth should strengthen psychological care. Psychological care after cesarean section and concern about the newborn health will plague the whole of the puerperium. At this stage, effective communication and notification are very important. Family care and her husband's company are the key of treatment. Many iatrogenic PTBs were the results of cesarean sections in China. Multiple pregnancy, HYP, and PP have been the common indicators for cesarean section. The cesarean rate has increased in China, up to $34.9 \%$ in 2014 , with geographic variations (43). It is well-known that cesarean section may result from non-medical etiological factors in China. If the cesarean section can be lowered, the rate of iatrogenic PTB would be decreased, and consequently, the total number of PTBs would be reduced.

\section{Limitations and strengths}

This study had some limitations. Clinical data were collected from the medical records of one hospital, which could lead to overlooking some unknown risk factors related to adverse pregnancy outcomes associated with mothers and newborns. Many previous studies have reported that maternal body mass index pre- and mid-pregnancy, economic status were important covariates; thus, our observations could be biased. But in the Wuxi, more pregnancy body weight is increase is normal.so in this area, the maternal body mass index preand mid-pregnancy influence is small. Little records about maternal environments and behaviors result in biases, which could have adversely affected data quality.

Despite the limitations, some strengths emerged in our study. It is only a few studies investigating the association between pregnancy complications and PTB with an age stratification. Furthermore, more adverse effects on PTB 
were observed in two groups, the advanced age, and the child-bearing age, providing a better understanding of the causes of poor fertility outcomes.

\section{Conclusions}

Our study demonstrated the incidence of PTB and identified several high factors associated with $\mathrm{PTB}$ in China. PP, PROM, and ICP increased the risk of PTB. The pregnancy complication may account for the Iatrogenic PTB, and the effects will increase the frequency of Iatrogenic PTB, especially in advanced-age pregnant women, because the two-child policy gathers momentum in China. Among all the risk factors, hypertension diseases in pregnancy, premature PROM, and PP were observed as the independent key factors for iatrogenic PTB. The prudent assessment and prudent management strategy of such pregnancies is required, as PTB has short-long term neonatal consequences. Pregnancy complications were associated with adverse birth outcomes. Further studies should identify how pregnancy complications can be avoided to have optimal pregnancy outcomes. Based on the very high rate of iatrogenic $\mathrm{PTB}$, we need to be continually reinforced and rethink our strategies to avoid unnecessary medical interventions. Active treatment and control of maternal primary disease, give some medicine to control of maternal blood pressure; reduce the rate of maternal first cesarean section will effectively remove the etiology of pathological preterm birth, this method will achieve the purpose of prevention and early intervention. If the incidence of a cesarean section can be lowered, the rate of iatrogenic PTB would be decreased, and consequently, the total number of PTBs would be reduced.

\section{Acknowledgments}

Funding: This work was supported by the National Natural Science Foundation of China (81671473), Medical Science and Technology Projects of Jiangsu Province (BE2016633), Scientific Research Project of Health Commission of Jiangsu Province (LGY2018015), and the Top Talent Project of Wuxi Municipal Health and Family Planning Commission (BJ2020081), and the New York State Research Foundation for Mental Hygiene (914-3280, 914-3257).

\section{Footnote}

Reporting Checklist: The authors have completed the STROBE Checklist. Available at https://dx.doi. org/10.21037/tp-21-458

Data Sharing Statement: Available at https://dx.doi. org/10.21037/tp-21-458

Conflicts of Interest: All authors have completed the ICMJE uniform disclosure form (available at https://dx.doi. org/10.21037/tp-21-458). The authors have no conflicts of interest to declare.

Ethical Statement: The authors are accountable for all aspects of the work, ensuring that questions related to the accuracy or integrity of any part of the work are appropriately investigated and resolved. All procedures performed in this study involving human participants were in accordance with the Declaration of Helsinki (as revised in 2013). The study was approved by the Research Ethical Committee of Nanjing Medical University (No. 2019-02-0102-01) and informed consent was taken from all the patients.

Open Access Statement: This is an Open Access article distributed in accordance with the Creative Commons Attribution-NonCommercial-NoDerivs 4.0 International License (CC BY-NC-ND 4.0), which permits the noncommercial replication and distribution of the article with the strict proviso that no changes or edits are made and the original work is properly cited (including links to both the formal publication through the relevant DOI and the license). See: https://creativecommons.org/licenses/by-nc-nd/4.0/.

\section{References}

1. Gomez-Lopez N, Romero R, Plazyo O, et al. Preterm labor in the absence of acute histologic chorioamnionitis is characterized by cellular senescence of the chorioamniotic membranes. Am J Obstet Gynecol 2017;217:592.e1-592.e17.

2. Blencowe H, Cousens S, Chou D, et al. Born too soon: the global epidemiology of 15 million preterm births. Reprod Health 2013;10 Suppl 1:S2.

3. Vogel JP, Chawanpaiboon S, Watananirun K, et al. Global, regional and national levels and trends of preterm birth rates for 1990 to 2014: protocol for development of World Health Organization estimates. Reprod Health 2016;13:76.

4. Chen C, Zhang JW, Xia HW, et al. Preterm Birth in China Between 2015 and 2016. Am J Public Health 2019;109:1597-604.

5. Zhao X, Chen Y, Qiu G, et al. Reducing preterm births in 
China. Lancet 2012;380:1144-5; author reply 1145.

6. Leung MP, Thompson B, Black J, et al. The effects of preterm birth on visual development. Clin Exp Optom 2018;101:4-12.

7. Kinzler WL, Prasad V, Ananth CV, et al. The effect of maternal thrombophilia on placental abruption: Histologic correlates. J Matern Fetal Neonatal Med 2009;22:243-8.

8. Scully M, Thomas M, Underwood M, et al. Thrombotic thrombocytopenic purpura and pregnancy: presentation, management, and subsequent pregnancy outcomes. Blood 2014;124:211-9.

9. Wang CH, Yang CY, Lien R, et al. Prevalence and independent risk factors for hearing impairment among very low birth weight infants. Int J Pediatr Otorhinolaryngol 2017;93:123-7.

10. Blencowe H, Cousens S, Oestergaard MZ, et al. National, regional, and worldwide estimates of preterm birth rates in the year 2010 with time trends since 1990 for selected countries: a systematic analysis and implications. Lancet 2012;379:2162-72.

11. Zou L, Wang X, Ruan Y, et al. Preterm birth and neonatal mortality in China in 2011. Int J Gynaecol Obstet 2014;127:243-7.

12. He C, Liu L, Chu Y, et al. National and subnational allcause and cause-specific child mortality in China, 19962015: a systematic analysis with implications for the Sustainable Development Goals. Lancet Glob Health 2017;5:e186-97.

13. Wroblewska-Seniuk K, Greczka G, Dabrowski P, et al. Hearing impairment in premature newborns-Analysis based on the national hearing screening database in Poland. PLoS One 2017;12:e0184359.

14. Saigal S, Doyle LW. An overview of mortality and sequelae of preterm birth from infancy to adulthood. Lancet 2008;371:261-9.

15. Mwaniki MK, Atieno M, Lawn JE, et al. Long-term neurodevelopmental outcomes after intrauterine and neonatal insults: a systematic review. Lancet 2012;379:445-52.

16. Jansen PW, Tiemeier H, Looman CW, et al. Explaining educational inequalities in birthweight: the Generation $\mathrm{R}$ Study. Paediatr Perinat Epidemiol 2009;23:216-28.

17. Ogawa K, Urayama KY, Tanigaki S, et al. Association between very advanced maternal age and adverse pregnancy outcomes: a cross sectional Japanese study. BMC Pregnancy Childbirth 2017;17:349.

18. Lu MC, Chen B. Racial and ethnic disparities in preterm birth: the role of stressful life events. Am J Obstet Gynecol 2004;191:691-9.
19. Marleen S, Hettiarachchi J, Dandeniya R, et al. Maternal clinical predictors of preterm birth in twin pregnancies: A systematic review involving 2,930,958 twin pregnancies. Eur J Obstet Gynecol Reprod Biol 2018;230:159-71.

20. Hannam K, McNamee R, Baker P, et al. Air pollution exposure and adverse pregnancy outcomes in a large UK birth cohort: use of a novel spatio-temporal modelling technique. Scand J Work Environ Health 2014;40:518-30.

21. Martinelli KG, Garcia ÉM, Santos Neto ETD, et al. Advanced maternal age and its association with placenta praevia and placental abruption: a meta-analysis. Cad Saude Publica 2018;34:e0206116.

22. Ville Y, Rozenberg P. Predictors of preterm birth. Best Pract Res Clin Obstet Gynaecol 2018;52:23-32.

23. Wong-Taylor LA, Lawrence A, Cowen S, et al. Maternal and neonatal outcomes of spontaneously conceived pregnancies in mothers over 45 years: a review of the literature. Arch Gynecol Obstet 2012;285:1161-6.

24. Martinez NG, Niznik CM, Yee LM. Optimizing postpartum care for the patient with gestational diabetes mellitus. Am J Obstet Gynecol 2017;217:314-21.

25. Liu Y, Wang X, Zou L, et al. An analysis of variations of indications and maternal-fetal prognosis for caesarean section in a tertiary hospital of Beijing: A populationbased retrospective cohort study. Medicine (Baltimore) 2017;96:e5509.

26. Zhong N. Achieving Lower Preterm Birth Rates in China via Reductions in Iatrogenic Preterm Births. Am J Public Health 2019;109:1489-90.

27. Gilman-Sachs A, Dambaeva S, Salazar Garcia MD, et al. Inflammation induced preterm labor and birth. J Reprod Immunol 2018;129:53-8.

28. Shah A, Faundes A, Machoki M, et al. Methodological considerations in implementing the WHO Global Survey for Monitoring Maternal and Perinatal Health. Bull World Health Organ 2008;86:126-31.

29. Souza JP, Gülmezoglu AM, Vogel J, et al. Moving beyond essential interventions for reduction of maternal mortality (the WHO Multicountry Survey on Maternal and Newborn Health): a cross-sectional study. Lancet 2013;381:1747-55.

30. Goldenberg RL, Culhane JF, Iams JD, et al. Epidemiology and causes of preterm birth. Lancet 2008;371:75-84.

31. Frey HA, Klebanoff MA. The epidemiology, etiology, and costs of preterm birth. Semin Fetal Neonatal Med 2016;21:68-73.

32. WHO Expert Consultation. Appropriate body-mass index for Asian populations and its implications for policy and 
intervention strategies. Lancet 2004;363:157-63.

33. Koullali B, Oudijk MA, Nijman TA, et al. Risk assessment and management to prevent preterm birth. Semin Fetal Neonatal Med 2016;21:80-8.

34. Richards JL, Kramer MS, Deb-Rinker P, et al. Temporal Trends in Late Preterm and Early Term Birth Rates in 6 High-Income Countries in North America and Europe and Association With Clinician-Initiated Obstetric Interventions. JAMA 2016;316:410-9.

35. Gyamfi-Bannerman C, Fuchs KM, Young OM, et al. Nonspontaneous late preterm birth: etiology and outcomes. Am J Obstet Gynecol 2011;205:456.e1-6.

36. Hughes K, Kane SC, Araujo Júnior E, et al. Cervical length as a predictor for spontaneous preterm birth in high-risk singleton pregnancy: current knowledge. Ultrasound Obstet Gynecol 2016;48:7-15.

37. Zeitlin J, Szamotulska K, Drewniak N, et al. Preterm birth time trends in Europe: a study of 19 countries. BJOG 2013;120:1356-65.

38. Downes KL, Hinkle SN, Sjaarda LA, et al. Previous prelabor or intrapartum cesarean delivery and risk of placenta previa. Am J Obstet Gynecol 2015;212:669.e1-6.
39. Delnord M, Zeitlin J. Epidemiology of late preterm and early term births - An international perspective. Semin Fetal Neonatal Med 2019;24:3-10.

40. Wu J, Ma J, Bao C, et al. Pregnancy outcomes among Chinese women with and without systemic lupus erythematosus: a retrospective cohort study. BMJ Open 2018;8:e020909.

41. Teoh PJ, Ridout A, Seed P, et al. Gender and preterm birth: Is male fetal gender a clinically important risk factor for preterm birth in high-risk women? Eur J Obstet Gynecol Reprod Biol 2018;225:155-9.

42. Kietpeerakool C, Lumbiganon P, Laopaiboon M, et al. Pregnancy outcomes of women with previous caesarean sections: Secondary analysis of World Health Organization Multicountry Survey on Maternal and Newborn Health. Sci Rep 2019;9:9748.

43. Li HT, Luo S, Trasande L, et al. Geographic Variations and Temporal Trends in Cesarean Delivery Rates in China, 2008-2014. JAMA 2017;317:69-76.

(English Language Editor: J. Chapnick)
Cite this article as: Huang L, Chen Z, Li J, Chen Y, Yin K, Chen Y, Hu L, Zheng X, Zhou T, Zhu Y, Chen D, Zhong N. Iatrogenic factors contributed to the high rate of preterm birth in a community hospital. Transl Pediatr 2021;10(10):2602-2613. doi: $10.21037 / \mathrm{tp}-21-458$ 